\title{
A PROPERTY OF TORSION-FREE MODULES OVER LEFT ORE DOMAINS
}

\author{
ARTHUR VAN DE WATER
}

\begin{abstract}
It is well known that for an integral domain $A$, the property that a module is divisible if and only if it is injective is equivalent to the property that $A$ is a Dedekind domain. In this paper, it is shown that if $A$ is a left Ore domain, then a torsion-free left $A$-module is divisible if and only if it is injective.
\end{abstract}

Introduction. This paper will contain some results that grew out of an attempt to solve the following problem: Let $A$ be a ring with no zero divisors and let $E$ be a left $A$-module. When can $E$ be embedded in a minimal divisible left $A$-module?

The problem has been solved in the case where $A$ is a Dedekind domain with the result that any $A$-module can be embedded in a minimal divisible $A$-module. In the case where $A$ is an integral domain, then it has been shown that every torsion-free $A$-module has a minimal divisible extension. This paper will show that over a left Ore domain, a torsion-free left $A$-module is divisible if and only if it is injective. From this fact, it will follow that any torsion-free left $A$-module can be embedded in a minimal divisible left $A$-module.

This paper constitutes a portion of the author's doctoral dissertation written at the University of South Carolina. The author is deeply indebted to Dr. Edgar Enochs for his help and direction on this topic.

In what follows, all rings will contain a unit and all modules will be unitary left modules. The usual definitions of torsion-free, divisible and injective have been extended to left modules, but coincide with the usual definitions over commutative rings.

Theorem 1. If $A$ is a left Ore domain, then a torsion-free left $A$ module is divisible if and only if it is injective.

Proof. The proof of the sufficiency is identical with the proof in the case where $A$ is a commutative ring and will be omitted here. For the proof of the necessary condition, let $E$ be a torsion-free and divisible left $A$-module and consider the diagram

Received by the editors July 15, 1969 and, in revised form, October 20, 1969.

AMS Subject Classifications. Primary 1615, 1680; Secondary 1640.

Key Words and Phrases. Torsion-free modules, injective modules, injective hull, divisible modules, noncommutative rings, left quotient field, left Ore domain. 


$$
0 \rightarrow \underset{\downarrow}{a} \stackrel{a}{\stackrel{i}{\rightarrow} A}
$$

where $Q$ is a left ideal of $A$. Let $\alpha \in Q$ and consider $\phi(\alpha)$. If $\phi(\alpha)=0$ for every $\alpha \in Q$ then let $f \in \operatorname{Hom}(A, E)$ be such that $f: \beta \rightarrow 0$ for every $\beta \in A$. Trivially we see that in this case $f \mid Q=\phi$ and thus $E$ is injective. If $\phi(\alpha) \neq 0$, then since $E$ is divisible, there is an element $x \in E$ such that $\alpha x=\phi(\alpha)$. Let $f \in \operatorname{Hom}(A, E)$ be such that $f: 1 \rightarrow x$. We must show that $f \mid Q=\phi$. Let $\beta \in Q, \beta \neq 0$ and let $r, s \in A-\{0\}$ be such that $r \alpha=s \beta$. This is possible since $A$ is a left Ore domain [5]. Then $f(\alpha)=\phi(\alpha)$ so $r f(\alpha)=r \phi(\alpha)$ and this implies $f(r \alpha)=\phi(r \alpha)$. Thus $f(s \beta)=\phi(s \beta)$ so $s f(\beta)=s \phi(\beta)$ and $s(f(\beta)-\phi(\beta))=0$. Since $E$ is torsionfree, we get that $f(\beta)=\phi(\beta)$. Thus $f \mid a=\phi$ so $E$ is an injective left $A$-module.

THEOREM 2. If $A$ is a ring with no zero divisors, then the following are equivalent:

(a) $A$ is a left Ore domain.

(b) Every torsion-free left A-module has a torsion-free injective hull.

Proof. (a) $\Rightarrow$ (b). Let $E$ be a torsion-free left $A$-module and let $H(E)$ denote the injective hull of $E$. Suppose $x \in H(E), \alpha \in A$, and $\alpha x=0, \alpha \neq 0$. Let $B$ be the submodule of $H(E)$ generated by $x$ and let $\beta \in A, \beta \neq 0$. There exist elements $r, s \in A-\{0\}$ such that $r \alpha=s \beta$. Thus $r \alpha x=0$ so $s(\beta x)=0$ and this says that $\beta x \notin E$ since $E$ is torsionfree. Since $\beta x \in B$ for every $\beta \in A$ and since $\beta x \in E, \beta \neq 0$, we see that $B \cap E=0 . H(E)$ is an essential extension of $E$ so this implies that $B=0.1 \cdot x \in B$ so $1 \cdot x=x=0$. Thus $H(E)$ is torsion-free.

(b) $\Rightarrow$ (a). Suppose that every torsion-free left $A$-module $E$ has a torsion-free injective hull $H(E) . A_{s}$ ( $A$ considered as a left module over itself) is torsion-free, so $A_{s}$ has a torsion-free injective hull $H\left(A_{s}\right)$. Let $a, b$ be nonzero elements of $A$. Since $A$ is a domain, the map $f: x a \rightarrow x$ from $A a \rightarrow H\left(A_{s}\right)$ is a left $A$-homomorphism, so extends to a map $f^{\prime}: A \rightarrow H\left(A_{s}\right)$. Let $f^{\prime}(1)=a^{-1}$. Then $b a^{-1} \in H\left(A_{s}\right)$. There is some element $\beta \in A-\{0\}$ such that $\beta\left(b a^{-1}\right) \in A_{s}$, for otherwise $b a^{-1}$ would generate a submodule of $H\left(A_{s}\right)$ whose intersection with $A_{s}$ would be 0 , contradicting the fact that $H\left(A_{s}\right)$ is an essential extension of $A_{s}$. Let $\beta\left(b a^{-1}\right)=\alpha$. Then $\alpha \neq 0$ and we note that $\alpha\left(a a^{-1}\right)=\alpha$ so $(\beta b) a^{-1}=(\alpha a) a^{-1}$. Since $H\left(A_{s}\right)$ is torsion-free, $\alpha a=\beta b$ and we get that $A$ is a left Ore domain.

Corollary. If $A$ is a left Ore domain, then every torsion-free left $A$-module can be embedded in a minimal divisible left $A$-module. 


\section{REFERENCES}

1. N. Bourbaki, Algèbre, Chapitre 1, Hermann, Paris, 1958.

2. N. Bourbaki, Algèbre, Chapitre 2, Actualités Sci. Indust., no. 1236, Hermann, Paris, 1962. MR 27 \#5765.

3. H. Cartan and S. Eilenberg, Homological algebra, Princeton Univ. Press, Princeton, N. J., 1956. MR 17, 1040.

4. L. Levi, Torsion-free and divisible modules over non integral domains, Canad. J. Math. 15 (1963), 132-151.

5. O. Ore, Linear equations in non commutative fields, Ann. of Math. (2) 32 (1931), 463-477.

Winona State College, Winona, Minnesota 55987 